\title{
Les (f)acteurs extérieurs et la crise belge de 2010-2011
}

\author{
Pression de la Commission européenne et des \\ marchés financiers? Sortie de crise!
}

Fabrice Quinet

\begin{abstract}
Résumé/Abstract
[Français] Cet article définit le paradigme extérieur dans lequel les négociations pour la formation du budget 2012 de la Belgique se sont déroulées après plus de 500 jours de travail sur les questions institutionnelles. Nous explicitons les deux (f)acteurs extérieurs ayant débloqué des négociations à l'arrêt, et qui ont abouti seulement cinq jours après la démission de Mr. Elio Di Rupo, alors en charge de la formation du gouvernement. Nous démontrons d'abord que la Belgique était sous la menace d'une amende européenne de 710 millions d'euros dans le cadre la Procédure de Déficit excessif en l'absence d'un budget pour 2012. La problématique de la dette belge est située dans son contexte européen et nous analysons l'impact d'une hausse des taux d'intérêt sur une dette publique au travers du cas (du «spectre») grec. Nous expliquons ensuite les raisons politiques pour lesquelles la Belgique a été dégradée par l'agence de notation Standard \& Poor's, et comment ce deuxième fait a servi d'accélérateur à des négociations qui semblaient à nouveau dans l'impasse.

Mots-clefs: Crise politique belge (2007-2011), budget, dette publique, formation d'un gouvernement, marchés financiers, Procédure de Déficit excessif, agence de notation financière.

[English] This article describes and examines the external context in which the negotiations to compose the Belgian budget for 2012 took place, after more than 500 days of work on institutional issues. We show how two exterior circumstances unlocked in only five days the negotiations then on standstill after the resignation of Mr. Elio Di Rupo, then in charge of forming a cabinet. We first demonstrate that Belgium was threatened with a European fine of 710 million euros as part of the Excessive Deficit Procedure in the absence of a budget for 2012. This issue of the Belgian debt is set within the wider European context of the impact of a rise in interest rates on public debt. The Greek scenario - some say « spectrum » - is also evoked. In a second time, we shed light on the political reasons that explain why Belgium was downgraded by Standard \& Poor's, and we study how this downgrading dra-
\end{abstract}


matically accelerated the negotiations, which at that time seemed to have come to a dead end.

Keywords: Belgian political crisis (2007-2011), budget, public debt, cabinet formation, financial markets, Excessive Deficit Procedure, credit rating agencies.

\section{Introduction}

Après 526 jours de négociation pour former un gouvernement fédéral belge, dont près de cinq semaines de négociation budgétaire, le 21 novembre 2011, le Palais Royal publie un communiqué de presse dans lequel le Formateur du gouvernement, Elio Di Rupo, demande à être déchargé de sa mission en raison du «blocage des négociations budgétaires, sociales et économiques devant mener à la formation du gouvernement». Le communiqué insiste sur «la gravité de la situation actuelle et souligne que la défense de l'intérêt général de tous les Belges et les échéances européennes nécessitent une résolution très rapide de la crise politique ${ }^{1}$. Aussi, le Souverain tient-il sa décision en suspens.

Après une période d'optimisme suite à l'accord du 8 octobre 2011 visant une réforme institutionnelle, le Royaume replonge alors dans une période d'incertitude. Le politologue Pascal Delwit indique dans un entretien au quotidien La Libre Belgique que le pays «n'est pas loin d'être au bord [d'un] précipice dont on ne connaît ni la profondeur ni le contenu $»^{2}$; l'autre journal de référence en Belgique francophone, Le Soir, s'interroge dans les termes suivants : «La Belgique est-elle ingouvernable? $»^{3}$.

Pourtant, seulement cinq jours plus tard - et alors que la presse relatait « la volonté du gouvernement en affaires courantes de préparer [...] un début de budget afin de parer à toute éventualité catastrophique [...] si Elio Di Rupo et Cie devaient se crasher $[\mathrm{sic}] »^{4}-$ un accord budgétaire était trouvé après une réunion marathon de près 18 heures. Le $1^{\text {er }}$ décembre, un «Projet de Déclaration de Politique Générale » de 177 pages était publié ; le lendemain il était formellement accepté par les six partis ayant mené les négociations budgétaires (PS, SPa, CD\&V, cdH, MR et Open $\left.V_{L D}{ }^{5}\right)$. La Belgique avait enfin un gouvernement fédéral de plein exercice.

${ }^{1}$ http://www.monarchie.be/fr/actuel/agenda/archives/audience-du-formateur-

blocage-des-negociations

${ }^{2}$ La Libre Belgique, 23 novembre 2011.

${ }^{3}$ Le Soir, 23 novembre 2011.

${ }^{4}$ La Libre Belgique, 25 novembre 2011.

5 Respectivement parti socialiste francophone, parti socialiste néerlandophone, parti social-chrétien néerlandophone, parti démocrate-humaniste (anciennement socialchrétien) francophone, parti libéral francophone, et parti libéral néerlandophone. 
Pourquoi les négociations ont-elles finalement abouti alors que celles-ci semblaient inextricables ? Quels sont les facteurs qui ont poussé les négociateurs réticents à l'accord budgétaire ? Pour répondre à ces questions, c'est vers des facteurs exogènes au système politique belge qu'il faut se tourner.

Deux éléments importants ont eu une influence décisive sur le cours des négociations visant à aboutir à un accord budgétaire pour le restant de la législature 2010 - 2014. D'une part, la menace brandie par la Commission européenne - par la voix du Commissaire aux Affaires économiques et monétaires, Mr. Olli Rehn - que la Belgique se voie infligée une amende dans le cadre de la «procédure de déficit excessif ». D'autre part, la dégradation d'un cran de la note de la Belgique pour les emprunts à long terme par l'agence américaine de notation financière Standard\&Poor's (ci-après S\&P).

\section{La pression des institutions européennes}

Pour bien comprendre l'effet que ces deux événements survenus en dehors du microcosme des négociations belges ont eu sur le dénouement de ces dernières, il s'agit d'en comprendre les fondements. Ainsi, en ce qui concerne la pression des institutions européennes, un bref retour aux normes de fonctionnement de l'Union s'impose.

\subsection{Le Pacte de stabilité et de croissance}

Le Pacte de stabilité et de croissance (PSC) a été adopté lors du Conseil européen d'Amsterdam de juin 1997. Il prolonge l'effort de réduction des déficits publics engagé en vue de l'adhésion à l'Union économique et monétaire (UEM). Néanmoins, contrairement à la politique monétaire, la politique budgétaire demeure une compétence nationale. Aussi, le Pacte de stabilité et de croissance est-il un accord des États membres de la zone euro visant à coordonner leurs politiques budgétaires nationales afin d'éviter l'apparition de déficits budgétaires excessifs. Il impose aux pays de la zone euro de présenter à terme des budgets proches de l'équilibre ou excédentaires et, pour ce faire, use principalement de deux types de dispositions. La première est la concertation, soit une surveillance multilatérale des situations économiques et financières des États membres prévue à l'article $121 \mathrm{du}$ traité de Lisbonne (ex-article 103TCE). La seconde disposition est coercitive, elle concerne une procédure en cas de déficits excessifs, prévue à l'article 126 du traité (ex. Art.104 TCE) et accompagnée de sanctions en cas de manquements de l'État concerné. 


\subsection{La Procédure de déficit excessif (PDE) et la gouvernance économique de l'UE}

Cette procédure coercitive est déclenchée lorsqu'un État dépasse la valeur de référence de $3 \%$ entre le déficit public prévu ou effectif et le PIB aux prix du marché (Protocole $\mathrm{n}^{\circ} 20$ au TCE sur la procédure concernant les déficits excessifs), ou s'éloigne de l'objectif d'avoir une dette publique en dessous de $60 \%$ de son PIB (ou sur une trajectoire s'approchant de cette valeur à un rythme satisfaisant). Si l'une de ces deux valeurs n'est pas respectée, le Conseil pour les Affaires économiques et financières (Ecofin) reconnaît la situation de déficit excessif (Article 126/6) et adresse des recommandations (Article 126/7) à l'État concerné pour qu'il corrige cette situation. Si le pays ne prend pas d' «actions suivies d'effets » visant à mettre fin à l'une ou l'autre des infractions constatées, le Conseil peut prendre des sanctions à son encontre (Article 126/8 et 9).

Selon le règlement $(\mathrm{CE}) \mathrm{n}^{\circ} 1056 / 2005$, le Conseil fixe un délai permettant à l'État membre d'entamer des actions suivies d'effets en conformité avec ses recommandations. Si l'État n'applique pas lesdites actions, des sanctions sévères peuvent être prises par le Conseil à l'encontre du pays en infraction. Ces sanctions prennent d'abord la forme d'un dépôt sans intérêt auprès de l'UE. Le montant de ce dépôt comprend: un élément fixe égal à $0.2 \%$ du PIB ; un élément variable égal à un dixième de la différence entre le déficit (exprimé en pourcentage du PIB de l'année au cours de laquelle ce déficit a été jugé excessif) et la valeur de référence (3\%). Chacune des années suivantes, le Conseil peut décider de renforcer les sanctions en exigeant un dépôt supplémentaire jusqu'à un plafond de $0.5 \%$ du PIB. Ce dépôt peut être converti en amende si dans les deux années suivantes, le déficit excessif n'a pas, de l'avis du Conseil, été corrigé. La sanction financière est donc bien réelle.

La crise économique et financière a accru la pression sur les finances publiques des États membres et pas moins de vingt-trois d'entre eux ont fait l'objet d'une procédure de déficit excessif. Aussi, en décembre 2010 la Commission européenne a-t-elle présenté six propositions législatives concernant la gouvernance économique de l'Union européenne, appelées dans le jargon le «six-pack». Ces nouvelles mesures se composent de cinq règlements et ainsi que d'une directive et ont été approuvées par les vingt-sept États membres de l'Union européenne et le Parlement européen en octobre 2011. Elles ont comme objectif de favoriser la stabilité de l'économie européenne et de prévenir une nouvelle crise dans l'Union.

À partir du 13 décembre 2011, ce paquet législatif est entré en vigueur avec comme conséquences que les sanctions financières pourront être infligées de manière presque automatique aux États membres de la zone euro faisant l'objet d'une PDE qui ne se conformeraient pas aux recommandations que le Conseil leur a adressées. En effet, les sanctions financières prévues à l'article 126 du traité prévoyaient précédemment que le Conseil statue «à une majorité des deux tiers des 
voix de ses membres pondérés ». Or, le six-pack prévoit dorénavant que dans le cas où un État membre de la zone euro manquerait à ses obligations, le Conseil pourrait lui imposer une sanction financière sauf si une majorité qualifiée d'États membres s'y oppose. La procédure de vote a donc été inversée, et l'on parle dès lors de « majorité qualifiée inversée ». Cette nouvelle procédure «rend l'application des règles plus stricte et plus automatique et, partant, plus dissuasive et plus crédible [...] [et peut] être imposée à tout moment après le 13 décembre [2011]. » ${ }^{1}$

Notoirement, l'article 211 du Traité du Rome instituant la Communauté économique européenne (TCE) accorde trois rôles principaux à la Commission européenne «en vue d'assurer le fonctionnement et le développement du marché commun » : elle applique les dispositions du Traité ainsi que des dispositions prises par les institutions en vertu de celui-ci ; elle formule des recommandations ou des avis sur les matières qui font l'objet du traité ; elle dispose d'un pouvoir de décision propre et participe à la formation des actes du Conseil et du Parlement européen dans les conditions prévues au présent Traité.

C'est en vertu du troisième rôle que l'article 126 du Traité prévoit que la Commission «surveille l'évolution de la situation budgétaire et du montant de la dette publique dans les États membres en vue de déceler les erreurs manifestes. Elle examine notamment si la discipline budgétaire a été respectée ». Si le rapport entre le déficit public prévu ou effectif et le produit intérieur brut est supérieur aux 3\% prévus, elle peut estimer qu'il y a un déficit excessif dans un État membre ou qu'un déficit de la sorte risque de se produire. Dans ce cas, elle adresse un avis à l'État membre concerné et elle en informe le Conseil. C'est donc sur avis de la Commission que le Conseil reconnaît la situation de déficit excessif d'un État membre de la zone euro et qu'il décide de prendre des sanctions financières à l'encontre du pays concerné par la procédure de déficit excessif explicitée supra.

\subsection{La Belgique au cœur de la procédure de déficit excessif}

Le 7 octobre 2009, à la lumière de son rapport automnal concernant les prévisions économiques et monétaires des pays de la zone euro, la Commission européenne adoptait - conformément aux règles énoncées à l'article 104(3) du Traité sur l'UE un rapport marquant l'ouverture d'une procédure de déficit excessif à l'encontre de neuf pays, dont la Belgique, en raison d'un déficit budgétaire supérieur à $3 \%$ du PIB. En effet, contrairement aux données belges prévoyant un déficit général de 3,4\% du PIB en 2009, le rapport de la Commission estimait que le déficit de la

\footnotetext{
${ }^{1}$ Cabinet du Commissaire Rehn, MEMO/11/898, Bruxelles, 12 décembre 2011.
} 
Belgique «sera sensiblement plus élevé $(-5,9 \%)$ et qu'il ne sera pas proche de la valeur de référence de $3 \% »^{1}$.

La Commission motiva donc le mois suivant sa décision et proposa au Conseil des recommandations visant à mettre fin à la situation de déficit excessif de la Belgique. Le 2 décembre, en application de l'article 126(6), le Conseil Ecofin approuva le rapport de la Commission constatant le déficit excessif belge. En outre, en vertu du paragraphe 7 de l'article 126 et sur base du rapport de la Commission, la Conseil adopta des recommandations qu'il adressa au Royaume afin de «mettre un terme à la situation de déficit excessif en 2012 au plus tard [... et ce ...] d'une manière crédible et durable en prenant des mesures à moyen terme $»^{2}$. Le Conseil fixa la date limite du 2 juin 2010 pour que le gouvernement fédéral engage une action suivie d'effets pour la mise en œuvre desdites recommandations.

Le 13 juillet 2010, le Conseil approuvait un rapport intermédiaire de la Commission concernant l'évaluation des recommandations transmises aux États membres concernés par une procédure de déficit excessif. Ce rapport concluait que la Belgique avait pris des actions adéquates suivies d'effets visant à appliquer les recommandations du Conseil et que par conséquent, il n'était pas nécessaire de prendre des mesures supplémentaires - soit les sanctions financières - dans le cadre de la procédure de déficit excessif. Cependant, la PDE n'était pas abandonnée car le déficit belge était calculé à 4,1\% du PIB en 2010. Aussi, la Belgique restait-elle sous supervision budgétaire de la Commission, et ce jusqu'à ce que le Royaume ne réduise de manière durable son déficit budgétaire en deçà des $3 \%$ prévus par le Traité.

Durant les mois qui suivront, la Commission européenne et le gouvernement belge - en l'occurrence le premier ministre et le ministre des Finances ${ }^{3}$ - ont échangé plusieurs courriers non publics dans lesquels les autorités belges s'engageaient à suivre les recommandations du Conseil. La version actualisée du programme de stabilité belge 2011-2014 estimait un déficit 2012 de 2,8\% du PIB ${ }^{4}$ et la Commission européenne prit acte des engagements belges.

\footnotetext{
${ }^{1}$ « the deficit would however come out significantly higher $(-5,9 \%)$ and is not expected to be close to the $3 \%$ of GDP reference value ». Commission européenne, Belgium Report prepared in accordance with Article 104(3) of the Treaty, SEC(2009) 1268 final, 7 octobre 2009 , p.8.

${ }^{2}$ Conseil de l'Union européenne, «Recommandations du Conseil à la Belgique pour qu'il soit mis fin à la situation de déficit public excessif », 15754/09, Bruxelles, 30 novembre, pp.10-11.

${ }^{3}$ Entretien avec Steven COSTERS, Conseiller EcoFin de la Représentation permanente de la Belgique auprès de l'UE, Bruxelles, 13 janvier 2012.

4 Commission européenne, European Economic Forecast Automn 2011 - Member States, Belgium, Novembre 2011, Bruxelles, pp.83-84.
} 
Le 10 novembre, soit plus de sept mois après l'engagement officiel belge de réduire le déficit public à $2,8 \mathrm{du}$ PIB, et deux semaines après le début des négociations sur le budget, le Commissaire européen aux Affaires économiques et monétaires, le Finlandais Mr. Olli Rehn, organisait une conférence de presse dans le cadre de la publication du rapport «Prévisions d'automne 2011 ». Durant cette conférence de presse, Mr. Rehn déclara que «la Belgique était l'un des États membres qui ne semblent pas corriger son déficit excessif de manière structurelle et durable ${ }^{1}$ et invita la Belgique à suivre les recommandations du Conseil du 7 juin 2011 concernant le programme de stabilité de la Belgique pour 2011-2014, et en particulier sur le premier point qui stipulait que

« la Belgique [devrait] s'attacher, au cours de la période 2011-2012, à profiter du redressement de l'économie pour accélérer le rythme de correction du déficit excessif $[\ldots]$ pour réaliser un effort structurel moyen d'au moins $0,75 \%$ du PIB. Le déficit moyen [devant] ainsi être ramené nettement sous la valeur de référence de 3\% du PIB en 2012 au plus tard. $»^{2}$

Les estimations du rapport de la Commission européenne prévoyaient que le déficit belge allait croissant malgré les engagements pris par le Royaume. La Commission jugea que «les mesures permettant d'atteindre cet objectif n'ont pas encore été décidées et [que] des négociations sur le budget 2012 sont toujours en cours $»^{3}$. De même, le rapport soulignait que le fait que la Belgique dispose d'un gouvernement d'affaires courantes «joue probablement un rôle limitant l'accroissement des dépenses publiques ${ }^{4}$. Aussi, en raison des facteurs relevés par le rapport d'automne de la Commission européenne, les prévisions faites pour la Belgique furent basées sur un principe de «no-policy-change» et le déficit budgétaire fut estimé à 4,6\% du PIB en 2012.

\footnotetext{
${ }^{1}$ La Libre Belgique du 14 novembre 2011.

${ }^{2}$ Commission européenne, «Recommandation du Conseil concernant le programme de national de réforme de la Belgique pour 2011 et portant avis du Conseil concernant le programme de stabilité actualisé de la Belgique pour la période 2011-2014, 7 juin 2011, p.6.

3 « The caretaker government has reiterated Belgium's commitment to meet the deficit target of $2.8 \%$ of GDP in 2012. However, the measures that will allow the achievement of this objective have not yet been decided and negotiations on the 2012 budget were still underway at the cut-off date of this forecast. »Commission européenne, European Economic Forecast Automn 2011 - Member States, Belgium, Bruxelles, novembre 2011, pp.8384.

${ }^{4}$ « the Leterme cabinet is acting as a care-taker since its resignation in April 2010 (Belgium has not had a fully-fledged government at the federal level since 18 months), which limits its action to the so-called "current affairs", probably played a role in limiting the rise in public expenditure ». Idem.
} 
Le lendemain de la conférence de presse, le Commissaire Rehn écrivit à nouveau au gouvernement belge et l'enjoignit de mettre en œuvre les recommandations du Conseil visant un budget 2012 en dessous du seuil de 3\% du PIB. En effet, le « sixpack» entrait en vigueur le mois suivant et si aucun accord budgétaire (soit l'absence d'action suivie d'effets) n'était trouvé avant cette date, la Belgique risquait une amende estimée à près de 710 millions d'euros !

La publication dans la presse belge d'éléments de la conférence du Commissaire européen ajoutait une pression supplémentaire aux négociations. Pourtant, Mr. Rehn ne « révélait» ces informations qu'à une opinion publique belge ignorante des complexes procédures européennes. Les négociateurs quant à eux n'ignoraient rien du danger avéré de l'amende de 710 millions d'euros car ils en furent informés à plusieurs reprises par la Représentation permanente de la Belgique (ci-après « RP ») auprès de $1^{\prime} U^{2}$. En outre, plusieurs notes rédigées par la RP soulignant l'importance du risque d'une telle amende ont également été transmises aux négociateurs ${ }^{3}$ et ceux-ci étaient donc conscients de l'entrée en vigueur du caractère presque automatique des sanctions financières prévues dans le cadre de la réforme de la procédure de déficit excessif au 13 décembre du mois suivant.

Après d'aussi longues négociations infructueuses, aucun des six partis négociant le budget n'aurait pu prendre la responsabilité - fût-elle collective - que la Belgique soit le premier pays membre de l'Union européenne à être sanctionné dans le cadre de la PDE, ni que le Royaume soit condamné à payer une amende aussi importante dans un contexte de crise économique. En effet, si tel avait été le cas, il est très probable que des élections anticipées auraient dû être convoquées et que les citoyens belges auraient sévèrement sanctionné les partis responsables de cet échec. Ainsi, ni les partis flamands, ni les partis francophones participant aux négociations n'avaient intérêt à prendre ce risque démesuré pour l'avenir du pays... et le leur. Par conséquent, il ne restait que quatre semaines aux négociateurs pour conclure le budget 2012, et ce, quels que soient les états d'âme des partis associés aux négociations.

\section{La pression des Agences de notation financière et des marchés financiers}

Tout comme il était nécessaire de rappeler les fondements normatifs sous-tendant les pressions des institutions européennes, il est maintenant intéressant de passer brièvement en revue le contexte et les mécanismes dans lesquels évoluent les

\footnotetext{
${ }^{1}$ Entretien avec Steven Costers, op. cit.

2 Idem.

${ }^{3}$ Ibidem.
} 
agences de notation, l'une d'entre elles - Standard \& Poor's - ayant eu un impact direct sur le dénouement des négociations.

\subsection{Les besoins de financement des dettes publiques et les marchés financiers}

\subsubsection{Les dettes publiques et leur financement}

La dette publique des États modernes est la conséquence de déficits budgétaires résultant de recettes inférieures aux dépenses, et donc de soldes budgétaires négatifs. Ce solde budgétaire se subdivise en deux éléments : le solde primaire d'une part, c'est-à-dire la différence entre les recettes et les dépenses de l'année hors paiement des intérêts de la dette, et d'autre part le paiement des intérêts sur la dette publique de l'année en cours.

Outre un effort à effectuer au niveau des dépenses ou des recettes, l'État doit en effet rembourser ses dettes et les intérêts de ces dernières. Pour ce faire, les États ont deux choix : émettre de la monnaie ou emprunter sur les marchés financiers. La première option est appelée «monétisation de la dette ( (ou plus communément «faire tourner la planche à billet»): le pays rembourse sa dette par une monnaie qu'il crée lui-même. Cependant, cette méthode a été abandonnée par les pays développés en général et les pays européens en particulier depuis plusieurs décennies car, dans la perspective de l'Union monétaire, les États membres de l'UE ont amendé les statuts de leur banque centrale et opté pour l'indépendance de l'institution monétaire. De plus, l'article 101 du traité de Lisbonne interdit aux banques centrales de la zone euro et à la $\mathrm{BCE}$ « d'accorder des découverts ou tout autre type de crédit aux [...] autres autorités publiques [...] des États membres». Par conséquent, le recours aux «établissements privés de crédit »- c'est-à-dire les marchés financiers - est la seule alternative de financement pour les États européens membres de la zone euro qui ne veulent ou ne peuvent augmenter leurs recettes, ou diminuer leurs dépenses.

\subsubsection{L'évolution des dettes publiques}

Le financement traditionnel des dettes publiques a donc évolué vers un recours quasi exclusif au «marché », ce financement se faisant au travers de titres négociables, souvent des obligations. L'État est endetté de manière très majoritaire à taux d'intérêt fixe et les titres sont principalement émis à moyen et long terme. Ceci permet de limiter l'exposition de l'État au risque de liquidité en répartissant sur un plus grand nombre d'années la charge de refinancement de la dette. Les titres émis par l'État sont essentiellement détenus par des investisseurs institutionnels et ce sont 
les particuliers qui détiennent la majeure partie des dettes souveraines au travers de produits d'épargne (assurance-vie, SICAV, FCP). Cependant, l'internationalisation croissante de la dette des États développés progresse, notamment car la création de l'euro a conduit à un mouvement de diversification des portefeuilles' .

Ces dettes publiques ont considérablement augmenté à partir des années 1980. En moins de 30 ans, soit de 1970 à 1998, l'endettement de la zone euro par rapport à son PIB est passé de 36,6\% à 81,5\% ${ }^{2}$. En effet, dans les années 1980 et 1990, la croissance a continué à baisser, et ce alors que les taux d'intérêt des dettes souveraines augmentaient fortement, aggravant par là le poids de la charge de la dette des États. L'écart entre taux d'intérêt et taux de croissance grandissant, les États auraient dû dégager d'importantes recettes ne fût-ce que pour maintenir leur taux d'endettement ; ils n'ont pas été en mesure de le faire en raison de la baisse de la croissance et de choix politiques malheureux. Ce n'est qu'à partir de la fin des années 1990 que les taux d'intérêt ont baissé, grâce d'une part au passage de la monnaie de transaction des devises nationales à l'euro, et d'autre part au reflux de l'inflation de manière durable. Ainsi, l'écart entre taux d'intérêt et taux de croissance a diminué de manière très significative, permettant aux États de baisser de manière importante leurs taux d'endettement : c'est ainsi que l'endettement de la zone euro par rapport au PIB a ensuite diminué de près de dix points de base pour atteindre $71,8 \%$ en $2007^{3}$.

Néanmoins, la crise financière et économique de 2008 a provoqué une récession dans la majorité des pays industrialisés et poussé les grandes économies à opter pour des politiques de relance très coûteuses. Les pays développés se sont endettés à nouveau, et le taux d'endettement de la zone euro par rapport au PIB a crû de plus de vingt points en quatre années seulement pour atteindre $92,9 \%$ en $2010^{4}$ ! Heureusement pour les États européens, les taux d'intérêt n'ont cessé de diminuer en réaction à la crise financière et économique. En effet, la Banque centrale européenne n'a eu de cesse de diminuer son principal taux directeur, soit le taux d'intérêt appliqué aux opérations principales de refinancement de l'Eurosystème. Celui-ci

\footnotetext{
${ }^{1}$ Banque nationale de Belgique, "Rapport 2010 - évolution économique et financière », Bruxelles, février 2011, pp.99-100.

2 Voir le tableau «Engagements financiers bruts des administrations publiques en pourcentage du PIB » sur le site de l'OCDE, http://stats.oecd.org/Index.aspx?QueryId=32442\&lang=fr, consulté le 10 janvier 2012.

${ }^{3}$ Idem.

${ }^{4}$ Ibidem.
} 
passera d'un taux de 5,25\% en juillet 2008 à un taux de $1,75 \%$ en mai $2009^{1}$ - soit son taux historique le plus bas - et ce de manière à « favoriser la baisse [...] des taux à terme du marché monétaire [...], de contribuer à l'amélioration de la situation de liquidité sur d'importants compartiments du marché de la dette privée et d'assouplir les conditions de financement des banques et des entreprises. $»^{2}$

\subsection{Le spectre grec-Tensions sur les marchés des dettes souveraines européennes}

Pourtant, cette situation connaîtra une inflexion à partir de 2010, lorsque les taux sur les dettes souveraines de certains pays très endettés vont croître de manière importante et entraîner des mouvements spéculatifs aggravant cette tendance. Cette évolution à la hausse touchera plusieurs États de la zone euro, inélégamment appelés les «PIIGS » pour « Portugal, Italie, Irlande, Grèce et Espagne ». Les taux d'intérêt des obligations d'États à 10 ans auront dépassé les $10 \%{ }^{3}$ pour la Grèce, l'Irlande et le Portugal en juillet 2011 et ce sera Athènes qui sera la première et le plus sévèrement touchée.

De janvier 2010 à janvier 2012, le taux d'intérêt des obligations grecques à dix ans passera de $4,84 \%$ à $44,59 \%^{4}$, soit une augmentation de près de $1000 \%$ en seulement de deux ans ! Face à des taux d'intérêt très (!) élevés, Athènes se trouvera dans l'incapacité croissante d'émettre des titres «à un prix trop fort [car] non tenable sur le long terme $»^{5}$ selon les dires de Mr. Provopoulos, directeur de la Banque centrale hellénique. Aussi l'Union européenne et le Fonds monétaire international (FMI) ont-ils dû apporter une aide financière à la Grèce afin d'éviter un défaut de paiement qui menaçait de produire «des réactions en chaîne pour d'autres pays [de la zone euro], comme cela avait été le cas lors de la faillite de [la banque] Lehman Brothers » ${ }^{6}$. Cette intervention s'est accompagnée d'un plan d'austérité drastique visant à diminuer les déficits et à regagner la confiance des marchés : réduction de $10 \%$ des dépenses de l'État, diminution des salaires des fonctionnaires,

1 Voir le tableau «Key ECB interest rates» sur le site de la BCE, http://www.ecb.int/stats/monetary/rates/html/index.en.html consulté le 10 février 2012.

2 Banque centrale européenne, «Bulletin mensuel », Francfort-sur-le-Main, mai 2009, p.1.

${ }^{3} \mathrm{Au} 14$ juillet 2011, les taux de la Grèce étaient de 16,66\%, du Portugal de 12,87\% et de l'Irlande de 13,91\%. Voir site www.euronext.com, consulté le 14/01/2012.

${ }^{4}$ En date du 13/01/2012.Voir le site de la Bourse Euronext, consulté le 13 janvier 2012.

5 Propos recueillis dans Trends-Tendances, 8 mars 2010.

${ }^{6}$ Dans L'Expansion, 29 janvier 2010. 
gel des pensions, hausse de la TVA, hausse des taxations sur l'alcool, le tabac et le carburant et recul de l'âge de la retraite, entre autres. Face à cette politique de forte austérité, plusieurs grèves générales auront lieu, dont certaines particulièrement violentes: le 5 mai 2010, lors de la troisième grève générale, trois personnes trouveront la mort lors de l'incendie d'une banque par des cocktails Molotov. En plus des conséquentes retombées économiques, cette situation aura également d'importantes conséquences sociales. En effet, une étude parue en octobre 2011 dans le journal médical britannique Lancet relevait « des tendances très inquiétantes [comme] un doublement des cas de suicides, une hausse des homicides, une augmentation de 50\% des infections au virus HIV et des gens [... dont la ...] santé a empiré mais qui ne peuvent plus consulter de médecins même s'ils devraient le faire. ${ }^{1}$ Face à cet abîme économique et social, le spectre grec va commencer à hanter les États européens endettés.

\subsection{Le rôle des agences de notation dans les phénomènes spéculatifs sur les marchés, et le cas belge}

L'augmentation des taux d'intérêt grecs ne peut pas être uniquement expliquée par le risque accru lié à l'accroissement de la dette publique. En effet, le risque de défaut de remboursement de la Grèce n'était pas beaucoup plus élevé que précédemment pour les prêteurs, car la Banque centrale européenne (BCE) avait pris l'importante décision d'accepter «les titres grecs, quelle que soit leur notation, en garantie (collatéral) contre des prêts $»^{2}$. Ainsi, acheter des emprunts obligataires grecs était davantage risqué, mais ceux-ci n'étaient cependant pas les investissements spéculatifs que leur taux d'intérêt reflétait puisque ces obligations étaient « couvertes » de fait par la Banque centrale européenne. Ce qui précipite l'envolée des taux grecs, c'est la dégradation de sa note par les agences de notation financière.

\subsubsection{Les agences de notation financière et la notation des dettes souveraines}

Les agences de notation financière sont des sociétés privées spécialisées dans l'appréciation du risque de défaut de paiement lié à un titre de créance, comme notamment les obligations émises par les États. Les trois entreprises de notation les plus importantes sont américaines; il s'agit de Moody's, de Standard \& Poor's (S\&P) - qui se partagent chacune $40 \%$ des parts de marché - ainsi que de Fitch

\footnotetext{
${ }^{1}$ Étude citée dans France Soir, 10 octobre 2011.

${ }^{2}$ Le Monde, 4 mai 2010.
} 
Ratings, qui détient quant à elle $14 \%^{1}$ du marché. Ces agences analysent les données financières disponibles et synthétisent ces diverses informations sous la forme d'une «notation» qu'elles communiquent périodiquement au marché au travers de différents canaux comme la presse financière, le réseau d'information de la bourse ou des communiqués de presse. Cette notation a pour but de donner « une évaluation indépendante, objective et rigoureuse de l'aptitude ou de la capacité d'une entité économique à honorer aux échéances prévues ses obligations financières, c'est-àdire le remboursement du capital et des intérêts d'un emprunt » (KARIOTYS 1995, p.3). La notation financière est donc l'appréciation du risque de la solvabilité financière. En effet, dans un marché imparfait, il est coûteux et difficile pour un investisseur d'évaluer lui-même le risque de solvabilité d'un emprunteur. Aussi, afin de baisser les coûts de collecte de l'information et d'améliorer l'évaluation du risque d'un emprunteur, les acheteurs de titres de créance - soit les prêteurs - font appel à une agence de notation financière qui va sous-traiter cette analyse et cette appréciation ${ }^{2}$.

\subsubsection{Les conséquences d'une dégradation de note de dette souveraine}

L'influence des dégradations des notes souveraines sur l'augmentation des taux d'intérêt a été démontrée par plusieurs études. L'Autorité des Marchés Financiers de France (AMF) remarquait en 2006 que «les prix des actions réagissent plus fortement à la dégradation de la note ou à sa mise sous surveillance négative qu'à son relèvement ou à sa mise sous surveillance positive [... en particulier quand ...] l'environnement macroéconomique est défavorable » ${ }^{3}$. En outre, le Fonds Monétaire International a également analysé l'impact des annonces de notations souveraines sur les marchés et a conclu que "la dégradation de notes souveraines a statistiquement et économiquement des effets de contagion [...] sur les marchés financiers ce qui implique que les annonces des agences de notation peuvent propager l'instabilité financière » (AREZKI, CANDELON \& SY 2011, p.20) propice aux vagues spéculatives qui aggravent les tendances des marchés qui ne peuvent être corrigés par une « dévaluation interne » qui diminuerait mécaniquement le poids de la dette car les pays membres de la zone euro ne peuvent émettre de la monnaie et leurs Banques centrales ne peuvent racheter leurs dettes 4 .

1 The Washington Post, 23 novembre 2004.

${ }^{2}$ Les agences de notation financière ont été la cible de nombreuses critiques pour leur rôle dans la crise financière de 2007. Elles ont en effet bien noté les « subprimes ", qui se révéleront pourtant être des titres de type spéculatif.

3 AMF: L'impact de la notation, 31 janvier 2006, p.2.

${ }^{4} \mathrm{Il}$ y a des contre-exemples à cette logique : le Royaume-Uni et les États-Unis ont été dégradés et n'ont pas connu d'importantes hausses de leurs taux d'intérêt, mais ces 
Ce n'est donc pas un hasard si la forte croissance des taux d'intérêt sur les obligations de l'État grec est concomitante avec la dégradation de sa note de crédit à long terme, par les trois agences de notation financière, durant les dernières semaines du mois de décembre 2009. En outre, la dette souveraine grecque était placée « sous surveillance en perspective négative ». Une nouvelle dégradation de la Grèce rendrait sa note de type "spéculative ", et cette éventualité était désormais probable à court terme. L'annonce de cette dégradation eut comme conséquence de renforcer «les incertitudes autour de la dette publique grecque » (CHARTIER 2010, p.4) et marque le début de la spirale de la hausse des taux grecs. La Grèce, qui avait lancé deux emprunts en 2010 , l'un à six mois à un taux de 1,38\% et l'autre à douze mois à un taux de $2,20 \%$, devra verser, trois mois plus tard, des taux respectivement de $4,55 \%$ et de $4,85 \%$ !

\subsubsection{La dette publique belge sous pression des marchés financiers}

Au moment de la première vague d'adoption de l'Euro, la Belgique était le second État le plus endetté d'Europe après l'Italie et avant la Grèce, et ce «trio » était composé des seuls pays dont le taux d'endettement dépassait $100 \%$ du PIB, soit $119,5 \%$ pour la dette belge en 1999. Cependant, le gouvernement belge entreprenait alors une politique de rigueur sinon d'austérité et put ainsi réduire sa dette publique. De 2000 à 2007, la dette belge ne cessera de décroître : elle est passée de 113,6\% à 92,9\% du PIB, soit une baisse de plus de vingt points de pourcentage ${ }^{1}$. De même, après avoir culminé à 11,6\% du PIB en 1990, les charges d'intérêts de la Belgique ont reculé de manière continue. Cette tendance a participé de manière importante à la baisse de l'endettement belge qui a été «fortement liée à la diminution ininterrompue du taux d'intérêt de la dette publique en raison du refinancement à un taux plus faible des emprunts $»^{2}$. Et, malgré l'augmentation de la dette belge qui a suivi la crise économique de 2008, les taux d'intérêts sur la dette belge ont encore baissé en 2010 pour atteindre en août de cette année le chiffre de $2,87 \%$, soit «le niveau le plus bas depuis l'après-guerre ${ }^{3}$.

Cependant, au contraire de ses voisins directs, les taux d'intérêt sur les obligations de la Belgique vont progressivement augmenter au cours de l'année 2011. Le taux d'intérêt sur les obligations belges à dix ans - les obligations à long terme

deux pays peuvent émettre de la monnaie et leurs banques centrales peuvent racheter des obligations émises par les administrations publiques, contrairement aux pays membres de la zone euro.

${ }^{1}$ Voir tableau OCDE, op. cit.

${ }^{2}$ Banque Nationale de Belgique, op.cit., p.99.

${ }^{3}$ Belga, «Le taux d'intérêt des obligations belges historiquement faible », 24 août 2010. 
représentent $89 \%$ des besoins de financement en $2011^{1}$ - qui était de 3,99\% en décembre 2010 aura progressé de plus d'un point lorsque les négociations budgétaires $^{2}$ commenceront au cours du mois d'octobre de l'année suivante ${ }^{3}$. Cette lente augmentation des taux s'explique par la durée anormalement longue des négociations en vue de former en gouvernement - la plus longue du monde - qui détériorera l'image de la Belgique perçue comme « un pays n'ayant pas les autorités politiques à même de prendre des mesures correctrices fondamentales » ${ }^{4}$. Aussi la hausse des taux s'est-elle accélérée durant le mois de novembre à mesure que les négociations sur le budget se prolongeaient. Trois jours seulement après que le Formateur eût démissionné et que les négociations budgétaires furent suspendues, les taux d'intérêt à dix ans augmenteront de vingt-cinq points de base à 5,74\%, soit le plus haut niveau depuis onze ans.

\subsubsection{Standard \& Poor's dégrade la note souveraine à long terme de la Belgique, et un accord est trouvé}

En dépit des taux élevés, l'agence fédérale belge pour la dette devait émettre deux milliards d'euros le 28 novembre 20115 pour financer le déficit budgétaire. Face à ces taux, le gouvernement belge en affaires courantes décida, le 23 novembre, d'émettre des bons du Trésor accessibles aux particuliers à des taux inférieurs à ceux du marché dans l'espoir de diminuer l'impact de la hausse des taux sur la charge d'intérêt de la Belgique. Mais seulement deux jours après l'annonce de l'émission de ces bons d'État belges, l'agence de notation financière Standard \& Poor's dégradait d'un cran la note souveraine à long terme de la Belgique, désormais notée AA contre AA+ auparavant. Dans un communiqué de presse émis à $18 \mathrm{~h} 30$ le vendredi 25 novembre 2011 - alors que, pour rappel, le Formateur était toujours démissionnaire - l'agence américaine expliquait sa décision de dégrader la Belgique en raison «de l'impasse des principaux partis politiques quant à une stratégie

\footnotetext{
${ }^{1}$ Agence Fédérale de la Dette: Besoins de financement et plan de financement 2012, 12 décembre 2011, p.5.

${ }^{2}$ La crise politique belge de 2010-2011 concernait la formation d'un gouvernement, mais il importait que les partis à la négociation s'entendent d'abord sur les réformes institutionnelles à poursuivre une fois le gouvernement formé, ensuite sur la politique budgétaire à mettre en œuvre, et enfin sur les orientations à donner dans plusieurs champs politiques (immigration, sécurité, etc.).

3 Voir les statistiques du taux de rendement à long terme des titres émis par les pouvoirs publics belges sur le site de la Banque Nationale de Belgique, consulté le 12 janvier 2012.

${ }^{4}$ Entretien avec E. de Callataÿ, dans La Libre Belgique du 8 août 2011.

${ }^{5}$ Agence Fédérale de la Dette, op.cit,, p.5.
} 
concernant la manière d'atteindre un déficit budgétaire de $2,8 \%$ en 2012 [...] depuis les élections de juin 2010 [...] ce qui pourrait réduire les capacités des autorités [belges] à répondre aux problèmes de ses finances publiques $»^{1}$. En outre, $\mathrm{S} \& \mathrm{P}$ prévoyait «qu'une augmentation des taux d'intérêt [...] ne peut être exclue $[\ldots]$ nécessitant davantage de mesures pour réduire le déficit de l'État $»^{2}$. Par ailleurs, l'agence plaça la note de la Belgique sous perspective négative «en raison des risques de croissance de la dette publique liés à l'impasse politique ${ }^{3}$. Le risque d'une seconde dégradation à court terme était donc explicite.

Les dispositions réglementaires actuelles prévoient la notification d'une notation à l'entité notée douze heures avant sa publication ${ }^{4}$; aussi la Belgique - et tout logiquement les négociateurs - a-t-elle été prévenue de sa dégradation par S\&P durant la matinée du vendredi 25 novembre. Les négociateurs étaient donc conscients de deux éléments lors de cette veille de week-end. Premièrement, la Belgique risquait une amende de plus de 700 millions d'euros si les négociations budgétaires n'aboutissaient pas avant le 13 décembre. Il ne leur restait donc plus que deux semaines pour trouver un accord. Deuxièmement, les taux sur les obligations belges qui n'avaient cessé d'augmenter étaient susceptibles de crôitre d'une manière telle que tout accord budgétaire serait de toute façon mis en péril par l'incapacité du pays à se financer à des taux supportables. Comme la dégradation belge est intervenue le vendredi soir - alors que les bourses européennes avaient fermé - la fenêtre d'ouverture s'étalant jusqu'au 13 décembre s'est réduite au week-end, car un accord devait être trouvé avant lundi matin, date de la réouverture des bourses. Par conséquent, aussi bien le Formateur démissionnaire que les négociateurs des cinq autres partis étaient-ils « condamnés » à un trouver un accord endéans les quarantehuit heures.

De fait, «c'est dans la famille libérale que les réticences à l'épure budgétaire sont les plus grandes $»^{5}$, et spécialement entre le PS et l'Open VLD pour lequel

\footnotetext{
${ }^{1}$ «For 2012, negotiations among the main political parties regarding a precise strategy on how to achieve a budget deficit of $2.8 \%$ of GDP have stalled [...] followed the June 2010 general election ». S\&P (2011): Rating on Belgium lowered to AA on financial sector risks to public finances, pp.3-4.

2 « We believe that a lasting increase in interest rates [...] cannot be excluded,would require further measures to reduce the primary balance ». Idem.

${ }^{3}$ " Ongoing risks to the government debt trajectory [...] due to protracted political uncertainty. We could lower the ratings further $»$. Idem.

4 Commission européenne, Proposition de règlement du Parlement européen et du Conseil modifiant le règlement (CE) $n^{\circ}$ 1060/2009 sur les agences de notation de crédit, novembre 2011, Bruxelles, p.11.

${ }^{5}$ La Libre Belgique, 25 novembre 2005.
} 
«plusieurs points ne passent pas ${ }^{1}$ en particulier en ce qui concerne la dégressivité des allocations de chômage ainsi que l'allongement du «stage d'attente» pour bénéficier desdites allocations. Cependant, la situation précaire du Royaume commandait que les négociations reprennent afin d'éviter que le spectre grec ne prenne une forme belge. Ainsi, tous les négociateurs se sont réunis le soir même de la dégradation de la note de la Belgique pour « une séance de négociations au finish d'une longueur rare $» 2$. Le lendemain midi, à l'issue d'un bras de fer de 18 heures entre socialistes et libéraux, un accord sur le budget sera trouvé respectant l'équilibre entre gauche et droite car sans «il n'y aura pas de ponction supplémentaire dans le domaine de la sécurité sociale [...] et des réformes structurelles seront engagées pour ce qui concerne le régime du chômage ${ }^{3}$. Cet accord - prélude à l'accord de gouvernement du 1er décembre - rassurera les marchés dans la capacité des partis politiques belges à s'entendre sur une stratégie concernant la manière de réduire le déficit. Et le lundi, les intérêts sur les obligations belges à dix ans sont retomberont à un taux de 5,5\%.

\section{Conclusions}

Après plus de cinq semaines de négociations budgétaires infructueuses pour arriver à un accord, les négociateurs se sont réunis à nouveau au Cabinet de la vicepremière ministre et ministre fédérale des Affaires sociales et de la Santé publique, Mme Onkelinx. Après avoir négocié durant « 18 heures au total pour boucler un accord à tout prix ${ }^{4}$, le samedi 26 novembre 2011 à midi, les négociateurs ont finalement abouti à un accord sur le budget 2012 ainsi sur que les trajectoires budgétaires pour les années 2012, 2013 et 2014, permettant normalement d'atteindre l'équilibre des finances publiques en 2015.

La rapidité avec laquelle l'accord sur le budget a été trouvé est liée à des facteurs exogènes au système politique belge. En comparaison avec les 531 jours de négociation sur les volets institutionnels, les 44 jours qui ont été nécessaires pour arriver à un accord budgétaire paraissent négligeables. La pression de la Commission européenne a d'abord créé un cadre temporel puisque les négociateurs étaient conscients qu'un accord sur le budget devait être trouvé avant le 13 décembre suivant. L'actuel Premier ministre belge, Mr. Elio Di Rupo, mentionnait d'ailleurs au lendemain de l'accord l'importance de «la pression de l'Union européenne [sur les négociations] qui n’a pas hésité à adresser à notre pays des

\footnotetext{
${ }^{1}$ Idem.

${ }^{2}$ La Libre Belgique, 28 novembre 2005.

${ }^{3}$ Le Soir (en ligne), 26 novembre 2011.

${ }^{4}$ Idem.
} 
menaces de sanctions ». La démission du formateur Di Rupo ne peut par conséquent s'expliquer que par une stratégie visant à faire aboutir un accord indispensable au regard des risques économiques et financiers encourus par la Belgique. En outre, la hausse des taux d'intérêt des obligations belges sur les marchés financiers, couplée à la dégradation de la note de la dette belge, a infligé aux négociateurs une pression telle que ces derniers furent forcés de parvenir à un accord avant l'ouverture des marchés deux jours plus tard. Le politologue Dave Sinardet notait à ce propos dans un entretien accordé à La Libre Belgique le surlendemain de l'accord sur le budget que « la dégradation de la note de la Belgique et la hausse des taux d'intérêt dans la zone euro ont poussé les négociateurs à conclure au plus vite ${ }^{1}$. Ainsi, ces éléments, par la pression qu'ils ont provoquée, sont les véritables catalyseurs de l'issue positive des négociations. L'éditorialiste et chef du service politique du quotidien flamand Het Laatste Nieuws, Mr. Luc Van der Kelen, résumera cruellement le dénouement de la crise politique belge par ces mots: «l'accord n'a pas été engrangé grâce à la volonté et au sens de l'État des négociateurs, mais parce que les marchés financiers et l'Europe ont mis le couteau sous la gorge de la Belgique $»^{2}$.

En effet, les négociateurs faisaient partie d'une nouvelle génération de politiques lassés par «l'interminable gestation [des] anciens compromis à la belge ${ }^{3}$. Et c'est grâce aux (f)acteurs extérieurs que les négociateurs atténueront leurs exigences et redécouvriront les atouts d'un compromis à la belge - gage de la survie de la Belgique - qui «a permis de pacifier pendant des décennies des conflits communautaires et politiques dans le pays ${ }^{4}$.

\section{Références bibliographiques}

\section{Articles :}

AREZKI R., CANDElON B., SY A. (2011): «Sovereign rating news and financial markets spillovers : Evidence from European debt crisis ». In IMF working papers.

CHARTIER J. (2010), Rapport sur la Commission des Finances, de l'Économie générale et du Contrôle Budgétaire sur le projet de loi de régulation bancaire et financière. Paris: Rapport de l'Assemblée nationale française.

\footnotetext{
${ }^{1}$ La Libre Belgique du 28 novembre 2011.

${ }^{2}$ Cité dans La Libre Belgique du 29 novembre 2011.

${ }^{3}$ Le Vif L'express du 22 octobre 2010

${ }^{4}$ La Libre Belgique du 14 octobre 2010.
} 
KARIOTYS D. (1995): «Une nouvelle approche du risque ». In Revue Banque, p.3.

LEROY G. (2011): «Qui détient la dette publique ?». In Fondapol, p.16.

\section{Études :}

Agence Federale DE LA DetTE: Besoins de financement et plan de financement 2012. 12 décembre 2011.

AMF: L'impact de la notation. 31 janvier 2006.

BANQUE CENTRALE EUROPEENNE : Bulletin mensuel. 14 mai 2009.

BANQUE NATIONALE DE BelgiQue: Rapport 2010 - évolution économique et financière. Bruxelles, février 2011.

COMMISSION EUROPÉENNE: Belgium Report prepared in accordance with Article 104(3) of the Treaty SEC(2009) 1268 final. Bruxelles, , 7 octobre 2009.

COMMISSION EUROPEENNE: European Economic Forecast Automn 2011 - Member States, Belgium. Bruxelles, novembre 2011.

Commission europennne: Proposition de règlement du Parlement européen et du Conseil modifiant le règlement (CE) $n^{o}$ 1060/2009 sur les agences de notation de crédit. Bruxelles, novembre 2011.

COMMISSION EUROPEENNE: Recommandation du Conseil concernant le programme de national de réforme de la Belgique pour 2011 et portant avis du Conseil concernant le programme de stabilité actualisé de la Belgique pour la période 2011-2014. Bruxelles, 7 juin 2011.

CONSEIL DE L'UNION EUROPEENNE : Recommandations du Conseil à la Belgique pour qu'il soit mis fin à la situation de déficit public excessif - 15754/09. Bruxelles, 30 novembre 2011.

STANDARD \& POORS (2011): Rating on Belgium lowered to AA on financial sector risks to public finances.

\section{Journaux}

La Libre Belgique des 8 août et 14, 23, 25, 28 et 29 novembre 2011.

Le Soir, mercredi 23 novembre 2011.

La Dernière Heure du 29 novembre 2011.

Le Nouvel Observateur du 11 avril 2010. 
L'Expansion du 29 janvier 2010.

Le Figaro du 4 décembre 2008.

Le Monde du 4 mai 2010.

Le Vif-L'express du 22 octobre 2010.

France Soir du 10 octobre 2011.

The Washington Post du 23 novembre 2004.

Trends-Tendances du 8 mars 2010.

\section{Entretiens :}

Entretien avec Steven Costers, Conseiller EcoFin de la Représentation Permanente de la Belgique auprès de l'UE, Bruxelles, 13 janvier 2012.

\section{Sites internet :}

Site de la Banque Nationale de Belgique: www.bnb.be, consulté le 12/01/2012.

Site de la Bourse Euronext: www.Euronext.com, consulté le 13/01/2012.

Site de la Banque Centrale européenne : www.ecb.int, consulté le 10/02/2012.

Site du journal Le Soir: www.lesoir.be, consulté le 26/11/2011.

Site de l'Organisation de Coopération et de Développement Économiques: www.oecd.org, consulté le 10/01/2012.

Site du Palais Royal de Belgique: www.monarchie.be, consulté le 13/01/2012.

\section{Divers :}

DEPECHE BELGA: «Le taux d'intérêt des obligations belges historiquement faible », Bruxelles, 24 août 2010.

MEMO DU CABINET DU COMMISSAIRE REHN: MEMO/11/898, Bruxelles, 12 décembre 2011. 